\title{
L'économie comportementale au service de la santé
}

\author{
Yann Arnaud $^{1} \cdot$ Roxane Borgès Da Silva ${ }^{1,2,3}$ (D) Olivier Drouin ${ }^{3,4}$ \\ Received: 9 October 2020 / Accepted: 26 February 2021 / Published online: 19 March 2021 \\ (C) The Canadian Public Health Association 2021
}

\section{Résumé}

L'épidémie de COVID-19 nous impose des changements structurels profonds tant sur les plans économiques que sanitaires et les méthodologies classiques doivent être perfectionnées pour mieux adapter les décisions de santé publique. Dans ce cadre, l'économie comportementale, au croisement des sciences économiques et de la psychologie sociale, est un domaine incontestablement innovant en la matière. En effet, contrairement aux modèles classiques, cette discipline intègre les déterminants psychosociaux dans les modèles économiques pour mieux prédire les comportements des individus. Depuis une vingtaine d'années, la communauté scientifique utilise l'outil quantitatif de cette approche, l'économie expérimentale, dans de nombreux secteurs de la santé tels que la prévention, la promotion, les ressources humaines et la signalisation sociale. Les études menées ont apporté des solutions efficaces pour améliorer les bonnes pratiques sanitaires et sont des inspirations à ne pas négliger pour la lutte contre la COVID-19. Elles ont permis notamment de redonner une place centrale au comportement naturel de l'homme, de comprendre comment l'environnement socio-économique influence les individus et d'anticiper les réactions humaines pour adapter rapidement les politiques publiques.

\begin{abstract}
The COVID-19 pandemic is bringing about far-reaching structural changes on both the economy and public health, and conventional methodologies have to be fine-tuned to assist public health decision making. In this context, behavioural economics, which is situated at the crossroads between economics and social psychology, is an undeniably innovative field. In contrast with conventional models, the economic models of behavioural economics incorporate psychological and social determinants to produce more accurate predictions of individual behaviour. In the last 20 years, the scientific community has been using this approach's quantitative tool, experimental economics, in many areas of health, including prevention, promotion, human resources and social signage. Studies have come up with effective solutions that have improved best public health practices and provided sources of inspiration that should not be overlooked in the fight against COVID-19. They have allowed natural human behaviour to take a central role again, helped us to understand how the social and economic environment influences individuals, and enabled us to anticipate human reactions and so make faster adjustments to public policies.
\end{abstract}

Mots-clés Économie comportementale $\cdot$ santé publique $\cdot$ coronavirus $\cdot$ COVID-19 $\cdot$ prévention primaire

Keywords Economics, behavioural $\cdot$ Public health $\cdot$ Coronavirus $\cdot$ COVID-19 $\cdot$ Primary prevention

Roxane Borgès Da Silva

Roxane.borges.da.silva@umontreal.ca

1 Centre interuniversitaire de recherche en analyse des organisations (CIRANO), Montréal, Québec, Canada

2 Centre de recherche en santé publique, Montréal, Québec, Canada

3 École de santé publique de l'Université de Montréal, Montréal, Québec, Canada

4 Centre de recherche du CHU Sainte-Justine, Montréal, Québec, Canada

\section{Introduction}

L'expérience inédite de l'épidémie de COVID-19 nous impose des changements structurels profonds tant sur les plans économiques que sanitaires. Les liens entre les sciences sociales et la santé ont nourri de nombreuses recherches et la littérature en a largement bénéficié depuis une vingtaine d'années. Aujourd'hui, il existe une volonté croissante de dépasser les théories classiques pour mieux adapter les décisions de santé publique. L'économie comportementale, au croisement des sciences économiques et de la psychologie 
sociale, est un domaine incontestablement innovant. Pour analyser ces problématiques comportementales, les économistes et les psychologues sociaux ont recours à la méthode quantitative et empirique de cette approche : l'économie expérimentale.

\section{Économie comportementale et économie expérimentale}

L'économie comportementale se distingue de l'économie classique. En effet, les théories classiques reposent sur des axiomes simples où les individus sont rationnels, perspicaces et égoïstes. La maximisation de l'intérêt individuel conduit à l'intérêt général (Demeulenaere, 2015). Cependant, de telles hypothèses sont incohérentes dans un monde où les croyances subjectives et l'altruisme coexistent.

L'économie comportementale se dissocie également de la psychologie en intégrant un socle de rationalité. Les théories psychologiques ne convergent pas toujours quand il faut analyser le comportement individuel. L'expérimentation et les mises en situation en économie comportementale permettent de révéler les préférences des individus sur différents facteurs psycho-sociaux, tout en conditionnant les comportements économiques, ce que la psychologie seule ne peut démontrer. L'aversion au risque en contexte d'incertitude, les motivations intrinsèques ou extrinsèques (désir de bien faire ou d'être récompensé), le désir de coopération, la volonté de tenir compte des effets des actions sur autrui sont de nombreux éléments qui nous permettent de mieux comprendre et prédire la formation des comportements.

Dans les années quatre-vingt-dix, plusieurs expériences ont mis en évidence des anomalies dans le comportement des individus qui ne correspondaient pas aux théories classiques. La prévention en santé entraîne des coûts immédiats et des avantages retardés. Par conséquent, l'individu classique ayant des préférences pour le présent, est moins susceptible d'adhérer aux mesures préventives. Cependant, les individus élargissent leur horizon temporel. Chapman et Coups (1999) ont démontré qu'il existe une relation entre la préférence pour le futur et la vaccination contre la grippe. De plus, les individus ne sont pas égoïstes comme le suggère la théorie classique. Plusieurs études ont également montré l'importance de tenir compte de l'altruisme égoïste et de l'altruisme paternaliste dans l'analyse du comportement des individus. L'altruisme égoïste (physical or selfish externalities) indique que la consommation de soins de santé des autres a un effet sur le bien-être individuel. L'altruisme paternaliste (caring externalities) souligne le fait que les individus se soucient de la consommation des soins de santé des autres quand ils en ont besoin (Hurley, 2010). Ces éléments réfutent les hypothèses classiques et démontrent que calibrer des politiques publiques sans en prendre compte nous exporte dans un monde artificiel et biaisé.

L'objectif premier de l'économie comportementale est d'intégrer ces déterminants psychosociaux dans les modèles économiques pour mieux prédire les comportements des individus (Eber \& Willinger, 2005). L'économie expérimentale fait référence aux outils les plus communément utilisés en économie comportementale. Il s'agit d'expérimentations en laboratoire, en ligne, et sur le terrain. Ils prennent la forme d'expériences, d'enquêtes et de questionnaires où l'on interroge les individus dans un environnement précis et contrôlé. L'économie expérimentale permet de confirmer ou infirmer les résultats de la théorie classique en effectuant une comparaison avec l'expérimentation dans le monde réel (Eber \& Willinger, 2005). Enfin, des mécanismes de correction sont proposés aux institutions pour contrebalancer les défaillances comportementales individuelles. Il s'agit des stratégies de paternalisme libertaire (Thaler \& Sunstein, 2003) ou de nudges (Thaler \& Sunstein, 2009). Ces dernières corrigent le comportement individuel dans l'intérêt collectif à l'aide d'incitations monétaires ou non, de sanctions, de normes sociales et de stratégies de coopération.

L'économie comportementale et les outils associés en économie expérimentale ont été utilisés au sein de nombreuses disciplines, notamment les sciences politiques (DenantBoemont et al., 2008; Montmarquette, 2010). Dans le contexte unique de la COVID-19, il est important de comprendre les comportements des individus face à ce choc pour mieux adapter les réponses de politiques économiques et sanitaires.

\section{Les applications en santé publique}

Les outils de l'économie expérimentale ont été utilisés par la communauté scientifique dans différents domaines, notamment la prévention, la promotion et la signalisation sociale.

\section{Prévention et promotion}

Il s'agit de comprendre les comportements et les croyances propres aux individus pour mieux adapter les politiques de prévention. Les outils de l'économie expérimentale identifient les moyens encourageant l'adoption de bonnes pratiques individuelles face aux recommandations de santé publique.

Dillard et al. (2010) montrent que les interventions narratives et éducatives pour promouvoir le dépistage du cancer colorectal ont accru la perception du risque de la maladie et de l'intérêt pour le dépistage. Ces expérimentations sont pertinentes puisqu'elles entraînent des changements de comportement à long terme. En effet, les individus 
expérimentés ont indiqué que les obstacles au dépistage auraient moins d'impact lors d'une expérience future. Ils incitent également les autres individus à adopter ces bonnes pratiques par un mécanisme de diffusion.

D'autres expérimentations, plus simples à administrer et moins onéreuses, offrent également des résultats concluants pour réguler les actions des individus. Par exemple, des rappels via des messages texte ont significativement augmenté le taux de prise de médicaments pour le sida et le taux de vaccination pour la grippe (Hardy et al., 2011; Stockwell et al., 2012).

\section{La signalisation sociale : un outil de l'économie expérimentale au service de la santé}

Les individus ont tendance à se conformer aux comportements d'autrui. En effet, l'image est importante dans notre société et l'adoption par la majorité de certains comportements pousse les divergents à agir de même pour ne pas se sentir exclus. L'économie expérimentale montre qu'il est possible de normer les pratiques sanitaires au nom d'un groupe, d'une ville ou d'une société tout entière. Dans une petite ville italienne, les donneurs de sang atteignant un certain niveau de quotas de dons ont l'opportunité de recevoir une médaille et de voir leur nom apparaitre dans le journal local (Lacetera \& Macis, 2010). Cette expérience a drastiquement augmenté la fréquence des dons, ce qui démontre que les préoccupations liées à l'image sont importantes. Enfin, dans le cadre de la COVID-19, la distanciation sociale est moins perçue comme une menace liée au fait qu'une personne soit malade. Cette mesure est devenue un moyen de freiner la propagation virus dans l'intérêt de tous. Quelques semaines après le début de la pandémie, ne pas respecter les règles de distanciations sociales dans les lieux publics est mal perçu et devient même un comportement anti-social (O'Connell et al., 2020).

L'économie expérimentale a redonné une place centrale au comportement naturel de l'homme, souvent ignoré auparavant. L'environnement socio-économique et l'influence d'autrui entrainent les individus à se comporter différemment de la théorie standard. Ces études expérimentales apportent des solutions pour corriger ces déviations et sont des inspirations à ne pas négliger pour la COVID-19.

\section{Un atout pour la COVID-19}

Dans le cadre de la crise sanitaire actuelle, l'intérêt de l'économie expérimentale est double. Tout d'abord, elle permet de comprendre comment les individus vont réagir face à ce choc et suivre les recommandations. Ensuite, elle permet d'anticiper les prochaines vagues pour adapter rapidement les politiques publiques tout en garantissant une meilleure allocation des ressources.

La recherche expérimentale doit trouver des nudges motivant les individus à se conformer aux recommandations de santé publique et à la vaccination à long terme. L'adhésion aux recommandations est conditionnée par l'éducation et la prévention, mais cela ne suffit pas. Il faut également intégrer les variables des fonctions de réactions individuelles. Il s'agit alors d'inclure les déterminants cognitifs individuels (préférences sociales et vis-à-vis du risque) et socio-économiques (origines ethniques, le genre, le niveau d'éducation, la culture). Les méthodes expérimentales permettront de révéler le réel comportement des individus face aux consignes sanitaires, leur désir de coopérer, leur niveau d'aversion au risque ou encore leur volonté de tenir compte des externalités associées à leurs attitudes.

L'économie comportementale possède cette volonté de dépasser les limites théoriques et mettre en avant l'empirisme pour mieux s'adapter aux phénomènes sanitaires et anthropiques.

Remerciements Nous remercions chaleureusement Claude Montmarquette pour sa relecture et ses conseils avisés.

\section{Déclarations}

Conflit d'intérêts Les auteurs déclarent aucun conflit d'intérêts.

\section{Références bibliographiques}

Chapman, G. B., \& Coups, E. J. (1999). Time preferences and preventive health behavior: Acceptance of the influenza vaccine. Medical Decision Making, 19(3), 307-314.

Demeulenaere, P. (2015). Homo oeconomicus: enquête sur la constitution d'un paradigme: Presses universitaires de France.

Denant-Boemont, L., Malgrange, P., Masclet, D., \& Montmarquette, C. (2008). L'économie expérimentale comme outil d'aide à la décision. Economie prevision, (1), 1-6.

Dillard, A., Fagerlin, A., Dal, S. C., Zikmund-Fisher, B., \& Ubel, P. (2010). Narratives that address affective forecasting errors reduce perceived barriers to colorectal cancer screening. Social Science \& Medicine, 71(1), 45-52.

Eber, N., \& Willinger, M. (2005). L'économie expérimentale. Editions la Découverte.

Hardy, H., Kumar, V., Doros, G., Farmer, E., Drainoni, M.-L., Rybin, D., ... Stanic, A. (2011). Randomized controlled trial of a personalized cellular phone reminder system to enhance adherence to antiretroviral therapy. AIDS Patient Care and STDs, 25(3), 153-161.

Hurley, J. E. (2010). Health economics (Vol. 4). Toronto: McGraw-Hill Ryerson.

Lacetera, N., \& Macis, M. (2010). Social image concerns and prosocial behavior: Field evidence from a nonlinear incentive scheme. Journal of Economic Behavior \& Organization, 76(2), 225-237.

Montmarquette, C. (2010). L'économie expérimentale au service des politiques publiques: exemple des politiques de l'éducation. Idees economiques et sociales, (3), 24-28.

O’Connell, K., Berluti, K., Rhoads, S. A., \& Marsh, A. (2020). Reduced social distancing during the COVID-19 pandemic is associated with 
antisocial behaviors in an online United States sample. PLoS One, 16(1), e0244974.

Stockwell, M. S., Kharbanda, E. O., Martinez, R. A., Vargas, C. Y., Vawdrey, D. K., \& Camargo, S. (2012). Effect of a text messaging intervention on influenza vaccination in an urban, low-income pediatric and adolescent population: a randomized controlled trial. JAMA, 307(16), 1702-1708.
Thaler, R. H., \& Sunstein, C. R. (2003). Libertarian paternalism. American Economic Review, 93(2), 175-179.

Thaler, R. H., \& Sunstein, C. R. (2009). Nudge: Improving decisions about health, wealth, and happiness. Penguin.

Publisher's note Springer Nature remains neutral with regard to jurisdictional claims in published maps and institutional affiliations. 